\title{
Frailty is independently associated with 1-year mortality for elderly patients with non-ST- segment elevation myocardial infarction
}

\author{
Niklas Ekerstad, Eva Swahn, Magnus Janzon, Joakim Alfredsson, Rurik Löfmark, Marcus \\ Lindenberger, David Andersson and Per Carlsson
}

\section{Linköping University Post Print}

\section{Tweet}

N.B.: When citing this work, cite the original article.

Original Publication:

Niklas Ekerstad, Eva Swahn, Magnus Janzon, Joakim Alfredsson, Rurik Löfmark, Marcus Lindenberger, David Andersson and Per Carlsson, Frailty is independently associated with 1year mortality for elderly patients with non-ST-segment elevation myocardial infarction, 2014, European Journal of Preventive Cardiology, (21), 10, 1216-1224.

http://dx.doi.org/10.1177/2047487313490257

Copyright: SAGE Publications (UK and US): 12 month Embargo http://www.uk.sagepub.com/home.nav

Postprint available at: Linköping University Electronic Press http://urn.kb.se/resolve?urn=urn:nbn:se:liu:diva-99008 


\title{
Frailty Is Independently Associated With One-Year Mortality for Elderly Patients With Non-ST-Segment Elevation Myocardial Infarction
}

\author{
Niklas Ekerstad, MD, PhD (corresponding author) ${ }^{1,5}$ \\ Eva Swahn, MD, Professor ${ }^{2}$ \\ Magnus Janzon, $\mathrm{MD}, \mathrm{PhD}^{2}$ \\ Joakim Alfredsson, $\mathrm{MD}, \mathrm{PhD}^{2}$ \\ Rurik Löfmark, MD, Associate professor ${ }^{3}$ \\ Marcus Lindenberger, $\mathrm{MD}, \mathrm{PhD}^{4}$ \\ David Andersson, Statistician ${ }^{1}$ \\ Per Carlsson, Professor ${ }^{1}$
}

Affiliations:

The Center for Medical Technology Assessment/IMH, Linköping University ${ }^{1}$

Division of Cardiovascular Medicine, Department of Medicine and Health Sciences, Faculty of Health Sciences, Linköping University, Department of Cardiology UHL, County Council of Östergötland, Linköping $^{2}$

Department of Medical Ethics, LIME, Karolinska Institutet ${ }^{3}$

Department of Medicine, Ryhov County Hospital, Jönköping ${ }^{4}$

NU County Hospital, Trollhättan-Vänersborg-Uddevalla ${ }^{5}$

Corresponding author:

Niklas Ekerstad,The Center for Medical Technology Assessment/IMH, Linköping University, Sandbäcksgatan 7 , 58183 Linköping, Sweden

E-mail: niklas.ekerstad@liu.se

Tel. +46(0)13222000 or 0736249652 


\title{
Frailty Is Independently Associated with One-Year Mortality for Elderly Patients with Non-ST-Segment Elevation Myocardial Infarction
}

\begin{abstract}
Background: For the large population of elderly patients with cardiovascular disease, it is crucial to identify clinically relevant measures of biological age and their contribution to risk. Frailty is denoting decreased physiological reserves and increased vulnerability. We analyzed the manner in which the variable frailty is associated with one-year outcomes for elderly non-ST-segment elevation myocardial infarction (NSTEMI) patients.
\end{abstract}

Methods and Results: Patients aged 75 years or older, with diagnosed NSTEMI were included at 3 centers, and clinical data including judgment of frailty were collected prospectively. Frailty was defined according to the Canadian Study of Health and Aging Clinical Frailty Scale. Of 307 patients, 149 (48.5\%) were considered frail. By Cox regression analyses, frailty was found to be independently associated with one-year mortality after adjusting for cardiovascular risk and comorbid conditions (hazard ratio $4.3 ; 95 \% \mathrm{CI}, 2.4-7.8$ ). The time to the first event was significantly shorter for frail patients than for nonfrail (34 days; 95\% CI 10-58; $\mathrm{p}=0.005$ ).

Conclusions: Frailty is strongly and independently associated with one-year mortality. The combined use of frailty and comorbidity may constitute an important risk prediction concept in regard to cardiovascular patients with complex needs.

Clinical Trial Registration—http://www.clinicaltrials.gov. Unique identifier: NCT01049997

The most common diagnostic category for frail elderly patients with complex needs for healthcare is cardiovascular disease $(1,2)$. According to a scientific statement from the American Heart Association Council, the evaluation of comorbidity, frailty and functional status is crucial when elderly patients with non-ST-segment elevation myocardial infarction (NSTEMI) are treated (3).

Many randomised, controlled trials (RCTs) $(4,5)$ exclude elderly patients with specified severe comorbidities, which limits the generalisability of the results to patients in routine practice (3, 6-9). In particular there is a lack of data regarding the long-term outcomes for these patients (10). The present guidelines are based primarily on RCTs and systematic reviews, with focus on the treatment of defined organ-specific diagnoses (e.g. NSTEMI). This makes them difficult to apply to individual patients with multiple or severe comorbid conditions. The benefit of interventions can increase for elderly patients due to the increasing age-related cardiovascular risk. On the other hand, these patients are at higher risk of 
complications (e.g. bleedings) $(11,12)$. Adhering to guidelines in caring for elderly patients with many comorbid conditions has been stated to have potentially undesirable consequences, such as side effects from multiple drug regimens (13).

It has been emphasized that clinical priorities vary with life expectancy, since the benefits of preventive interventions accumulate over time whereas the harmful effects of many interventions are immediate. In discussing prognosis with the very elderly, clinicians could use prognostic indexes and take into account individual clinical factors rather than merely population-based averages (14). Similarly, the Swedish national guidelines for heart disease point out that the patient's biological age, i.e. biological status and expected length of life rather than the patient's chronological age, is crucial for decision making (15). However, there is very limited guidance in regard to how biological age should be estimated and how it influences the benefit-risk ratio of interventions.

Frailty denotes a multidimensional syndrome characterised by decreased physiological reserves and increased vulnerability $(16,19-22)$. Frailty stratification predicts a patient's need for institutional care and risk of death $(17,18)$. There is not a single accepted operational definition. Frailty instruments have thus far been used and validated mainly in a geriatric context. However, they have also been identified as being potentially relevant for cardiac patients in regard to risk stratification for elderly patients with cardiovascular disease (23-28). In a recent study we found frailty, as well as comorbid conditions quantified by the coronary artery disease (CAD)-specific index (29), to be independently associated with short-term outcomes for elderly patients with non-ST-segment elevation myocardial infarction (30).

Our aim in this study is to describe patients aged 75 years or older with NSTEMI, especially in regard to the variables cardiovascular risk, comorbidity, and frailty, and to analyse the manner in which frailty is associated with survival rate and one-year outcomes for these patients.

\section{Methods}

\section{Study Sample}


Between October 2009 and June 2010 we included evaluable patients aged 75 years or older with diagnosed NSTEMI treated at Linköping University Hospital and the county hospitals in Trollhättan (NÄL-Uddevalla) and Jönköping (Ryhov). The patients received care in one or more of the following hospital departments: cardiology, acute medicine, geriatrics, and other areas of internal medicine.

This is an observational study focusing on a study instrument (Clinical Frailty Scale) that has not been used previously to predict risk of outcomes for NSTEMI patients. It was therefore difficult to perform a power analysis regarding outcomes for frail versus nonfrail elderly patients. However, before the study we tried to estimate the expected percentages of outcome events (one month, ref. 30) for frail and non-frail NSTEMI patients aged 75 years or older. We approximated these two groups of patients with the percentages of outcome events of GRACE- (30\%) and VIGOUR (15\%) populations, aged 75 years or older, respectively (3).Given this rough estimation, and a chosen level of significance of $5 \%$ and a power of 80 $\%$, approximately 260 patients should be included.

\section{Data Collection and Variable Selection}

Patients aged 75 years or older with diagnosed NSTEMI according to their attending physicians were consecutively included.

The Canadian Study of Health and Aging Clinical Frailty Scale is a 7-point scale with good predictive validity (regarding death and need for institutional care) and prognostic power that relies on clinical judgment (17) (Figure 1). It is a global clinical measure of biological age, and it combines comorbidity, disability, and cognitive impairment. Before start of the study the study nurses underwent training regarding assessment of frailty. Then they individually assessed the frailty of 30 patients. An intraclass correlation test (31) showed that the interrater reliability regarding the study nurses' judgment of frailty was very good at the individual level (intraclass correlation 2-way random consistency [30 cases, 4 raters], single measure: $0.97 ; 95 \%$ confidence interval [CI], 0.94-0.98\%). An intraclass correlation coefficient of $>0.75$ has been defined previously as indicating a good degree of agreement between the raters (32).

\section{Figure 1}


If the inclusion criteria were fulfilled and the patient had given informed consent, evaluation of the patient's degree of frailty was based on bedside judgment regarding frailty and other clinical information, including the records in the patient file. If a patient was unable to give informed consent (without manifesting unwillingness to participate), but there was sufficient clinical information including the records in the patient file, evaluation of the patient's degree of frailty was based on this information only (i.e. without bedside judgment). A few patients who fulfilled the inclusion criteria while evidently not fulfilling any of the exclusion criteria were for some reason not evaluated during the hospital care episode. For these cases the evaluation of frailty was based on the records in the patient file and/or information obtained via a telephone call to the patient, after the patient had provided written consent via a letter. After permission from the hospital board was received, a computer-based screening of the hospital's diagnosis register was performed intermittently to detect potentially eligible but already discharged NSTEMI patients.

There were 2 exclusion criteria: (1) if the patient manifested unwillingness to participate or (2) if the patient was not evaluable because of communication problems and insufficient clinical information to enable a judgment of frailty.

Other demographic and clinical patient characteristics that were thought to be potential confounders when analysing the manner in which frailty is associated with one-year mortality, were the following: chronological age, sex, cardiovascular risk, myocardial infarction classification, ejection fraction, diabetes mellitus, previous myocardial infarction, and comorbidities. Cardiovascular risk was assessed according to the Fast Revascularization during Instability in Coronary artery disease II (FRISC II) score $(15,33)$, which takes into account the following parameters denoting high risk: positive biochemical markers (troponins), ECG signs of myocardial ischemia, previous myocardial infarction, diabetes mellitus, chronological age $>70$ years, male sex, and inflammatory activation. The presence of 3 or 4 of the aforementioned parameters implies moderate cardiovascular risk, whereas the presence of 5 or more implies high cardiovascular risk. The impact of the comorbid conditions on risk was quantified by the coronary artery disease (CAD)-specific index (29) (Figure 2). Echocardiography, ECGs, laboratory testing, and registration of anthropometric data were done according to routine practice.

Follow-up was done one month (first follow-up) and one year (second follow-up) after the time of inclusion via the patient files and the Causes of Death Register. The Linköping 
Academic Research Center (LARC) group at Linköping University Hospital performed study monitoring four times from study start until the second follow-up.

\section{Figure 2}

\section{Clinical Outcomes}

The primary outcome in this study was one-year mortality. The one-year composite outcome was the composite of death from any cause, myocardial reinfarction, revascularisation due to ischemia, hospitalisation for any cause, major bleeding, stroke/transient ischemic attack, and need for dialysis up to one year after inclusion. We studied time to first event according to the one-year composite outcome.

A bleeding was defined as major if one of the following was present: intracranial bleeding, retroperitoneal bleeding, blood transfusion, hemoglobin decrease $>3 \mathrm{~g} / \mathrm{dL}$ with overt cause, or hemoglobin decrease $>4 \mathrm{~g} / \mathrm{dL}$ without overt source (34)

Stroke and transient ischemic attack were defined according to a consensus statement (35). Myocardial infarction (MI), including peri-procedural MI, was classified according to the Joint European Society of Cardiology, American College of Cardiology Foundation, American Heart Association, andWorld Heart Federation Task Force consensus statement (36). The events were recorded by the study nurses and reviewed by the study investigators.

\section{Statistical Analysis}

Statistical analysis was performed via SPSS version 19.0 and SAS version 9.1.3. Categorical data were analyzed by use of the Fisher exact test or the chi-square test, and continuous data were compared with the Student $t$ test. The association of frailty with the primary composite outcome was examined by Cox regression models (37) adjusted for relevant prognostic variables (age, sex, previous myocardial infarction, ejection fraction, presence or absence of diabetes mellitus, cardiovascular risk, classification of myocardial infarction, and comorbidities [i.e. the score according to the CAD index]).

All 2-way interactions between frailty and the other independent variables were tested to validate the findings of the regression model. The regression model including possible statistically significant interactions was evaluated with a likelihood ratio test. All independent variables included in the models were analyzed for possible collinearity with a variance 
inflation factor test. Variance inflation factor values of $>2.5$ were considered to indicate collinearity (38).

We studied the time between entry into the study and a subsequent event by the use of survival analysis. To describe the survival times and the event-free times of the patients we used Kaplan-Meier curves.

\section{Ethics}

The study was conducted in accordance with the Declaration of Helsinki and the latest version of the Good Clinical Practice Guidelines. The study was undertaken after the protocol and its appendices had received full approval from the Independent Ethical Review Board in Linköping. The main ethical issue was how to get a proper informed consent from these elderly patients.

\section{Results}

\section{Baseline Characteristics}

Between October 2009 and June 2010 we included 307 evaluable patients aged 75 years or older with diagnosed NSTEMI.

Of these 307 patients, $149(48.5 \%)$ were considered frail according to the study instrument (5-7 on the scale), and $75(24.1 \%)$ were considered moderately or severely frail (6-7 on the scale).

The baseline characteristics are presented in Table 1. Frail patients were slightly older than nonfrail patients, with a mean age of 85 years versus 83 years $(P=0.0003)$, although the age range was similar in both groups. Frail patients presented with a greater burden of comorbidity, and they had higher CAD index and cardiovascular risk scores (all $P<0.05$; Table 1). Furthermore, $78.5 \%$ of the frail patients presented with one or more severe comorbid conditions compared with $43.0 \%$ of the nonfrail patients. The two groups did not differ significantly regarding other characteristics. Age was used as a continuous variable, whereas the others were used as categorical variables.

\section{Outcomes}

\section{Unadjusted One-Year Outcomes}


Of 307 patients 231 manifested one or more event, see Table 2. Of these 231 patients, 198 (86\%) manifested rehospitalisation as the first event. Of these 198 rehospitalisations 99 were due to a cardiovascular cause, and 99 were due to a non-cardiovascular cause. Among frail patients mortality was more prevalent than among nonfrail patients $(\mathrm{P}<0.0001$; Table 2$)$. Survival analysis is reported, Figure 4.

Frailty was significantly associated with increased fulfillment of the one-year composite outcome (nonfrail $n=109$ [69.0\%]; frail $n=122$ [81.9\%]; $p=0.01$ ). The main driver of the higher event rate among the frail patients was the higher mortality rate [frail 73 (49.0\%); nonfrail $20(12.7 \%) ; \mathrm{p}<0.0001]$. Of the frail patients 49 were judged to have died from a cardiovascular cause, 16 died from a non-cardiovascular cause; in 8 cases the cause of death was uncertain. Of the nonfrail patients 18 were judged to have died from a cardiovascular cause, 2 patients died from a non-cardiovascular cause.

Frail patients were less likely to be treated in intensive cardiac care units than nonfrail patients (nonfrail, $\mathrm{n}=85$ [53.8\%]; frail, $\mathrm{n}=52$ [34.9\%]; $\mathrm{P}<0.001$ ) during the index episode of care. Furthermore, frail patients were less likely to undergo coronary angiography than nonfrail patients (nonfrail, $\mathrm{n}=73$ [46.2\%]; frail, $\mathrm{n}=23$ [15.4\%]; $\mathrm{P}<0.0001$ ). Ninety-six of 307 patients underwent coronary angiography. Of these patients, 4 patients were moderately or severely frail, 19 were mildly frail, and 73 were nonfrail (Figure 3). Patients with severe comorbidity were less likely to undergo coronary angiography, whereas neither the cardiovascular risk score nor the CAD-specific index predicted the performance of coronary angiography

\section{Table 2}

\section{Figure 3}

\section{Adjusted One-Year Outcomes}

Frailty was independently associated with one-year mortality using Cox regression analyses after adjustment for the CAD index score and cardiovascular risk (hazard ratio 4.3; 95\% CI, 2.4-7.8; $\mathrm{p}<0.0001$; Table 3), as was ejection fraction (EF) (hazard ratio 2.5; 95\% CI 1.3-4.8, 
$\mathrm{p}=0.002$ ), cardiovascular risk (hazard ratio 5.4; 95\% CI 1.5-20.0; $\mathrm{p}=0.012$ ), and age (hazard ratio 2.7; 95\% CI 1.3-5.7; $\mathrm{p}=0.035)$. The independent association of frailty and mortality remained when adjusting for individual diseases known to cause death, i.e. diabetes mellitus, chronic heart failure, previous CVI/TIA, COPD and renal impairment in a new regression model (data not shown).

The CAD index score was not associated with one-year mortality. No collinearity was indicated between the independent variables, and there was no interaction that influenced the results.

No variable was independently associated with the one-year composite outcome using Cox regression analyses (hazard ratio 1,$3 ; 95 \%$ CI $0.87-1.8 ; \mathrm{p}=0.22$ ). The time to the first event was significantly shorter for frail patients than for nonfrail (34 days; 95\% CI 10-58; $\mathrm{p}=0.005$ ).

\section{Table 3}

\section{Figure 4}

\section{Discussion}

Our study demonstrates frailty to be independently and strongly associated with one-year mortality for elderly NSTEMI patients. In this study, nearly half of the population was frail. Frail patients were slightly older than nonfrail patients, and they manifested an increased burden of disease. Other variables that were independently associated with one-year mortality were age, cardiovascular risk and ejection fraction. Time to the first event was significantly shorter for frail patients than for nonfrail patients.

Our study is a multicenter, prospective observational trial with very few exclusion criteria. Since our aim was to describe a representative sample of elderly NSTEMI patients treated in clinical practice, including patients not treated in coronary care units and patients with secondary coronary ischemia (36), i.e. not only coronary care unit patients, the study design seems appropriate. In an earlier publication, frailty was reported to be independently associated with short-term clinical outcomes for elderly NSTEMI patients, including one- 
month mortality (30). To our knowledge, this is the first study to demonstrate frailty as strongly and independently associated with one-year mortality as well. We did this using an easily applied clinical measure of frailty, which was evaluated before the start of the study and was shown to have very good interrater reliability. The study was carefully monitored at four time points.

The trial did not have enough statistical power to properly analyse the manner in which frailty influences the benefit of coronary angiography and the possible invasive treatment that can follow. Because follow-up was done with the use of patient files and the Causes of Death Register, quality of life and burden of symptoms were not measured explicitly. However, rehospitalisation for cardiovascular causes indirectly indicates the burden of symptoms.

In most frailty studies a geriatric patient cohort has been studied rather than acute heart patients. In these studies frailty has been reported to be associated with long-term mortality, hospitalisation, and institutionalisation for geriatric patients $(18,19)$. In the one-month followup of this study it was indicated that frailty is independently associated with short-term outcomes for elderly NSTEMI patients (30). Our present study including the one-year followup shows that frailty is independently and strongly associated with one-year mortality. Furthermore, no other independent variable, with the exception of ejection fraction, cardiovascular risk and age, was associated with one-year mortality in adjusted analysis with the use of Cox regression analysis, which emphasizes the predictive strength of frailty.

No variable was significantly and independently associated with the one-year composite outcome, which may be an expected finding since as many as 231 of 307 patients had at least one event. However, the time to first event was significantly shorter for frail patients than for nonfrail patients. Rehospitalisation was by far the most common event.

The Canadian Study of Health and Aging Clinical Frailty Scale is based on clinical judgment and is relatively easily applied in a clinical context. There may be other measures of frailty that are more sensitive, but that are also more time-consuming and costly to administer. In our study, almost half of the population was frail. Thirty percent of a community-dwelling population of octogenarians has been estimated to be frail. More relevant in comparison with our study, the prevalence of frailty in an elderly population requiring cardiac care ranges from $27 \%$ to $63 \%$, depending on the classification scheme (39). 
Despite overlap between frailty and comorbidity, the distinction between the concepts has been stressed in other studies $(23,40)$. In earlier studies including patients with CAD and using the same CAD-specific index, 16 and $24 \%$ of the patients, respectively, presented with high scores $(10,24)$. In regard to the burden of comorbidity in our study, $32 \%$ of the patients presented with high CAD-specific index scores, which is not surprising because our study included older patients than in earlier studies. This may be one of the causes of the finding that the CAD-specific index score was not associated with one-year mortality.

Frail patients were less likely to be treated in intensive cardiac care units during the index episode of care than nonfrail patients, and, similarly, they were less likely to undergo coronary angiography. Frailty and severe comorbidity were strong negative predictors for performance of coronary angiography, whereas degree of cardiovascular risk did not influence the use of this measure. Clinical decision making for elderly NSTEMI patients seems to be based on factors other than the estimation of cardiovascular risk. This observation could be compared with recommendations in Swedish, European, and American heart guidelines, which rely primarily on cardiovascular risk. There are possible alternative interpretations of this finding. One could argue that if frail patients indeed benefit from interventions, more of these patients should undergo coronary angiography to improve outcomes. On the contrary, one could argue that frail NSTEMI patients should be treated in coronary care units to a lesser extent than today because they are not judged to benefit from specific coronary care. In fact, as many as $78.5 \%$ of the frail patients manifested one or more severe comorbid conditions (e.g., acute bleeding, severe renal insufficiency, severe anemia, or severe dementia). Many of these conditions could be considered potential contraindications to invasive procedures. Clearly, this matter needs further evaluation. However it would probably be questionable from an ethical viewpoint to perform an interventional study on the frailest patients.

Clinical priorities vary with life expectancy, since the benefits of preventive interventions accumulate over time whereas the harmful effects of many interventions are immediate. For very elderly patients with cardiovascular disease it is therefore important to identify clinically and individually relevant measures of biological age and their contribution to risk and life expectancy. The use of frailty may constitute an important and robust risk prediction concept in regard to cardiovascular patients with complex needs. 
In conclusion, our study indicates that frailty is independently and strongly associated with one-year mortality for elderly NSTEMI patients. In the one-month follow-up frailty was also independently associated with short-term outcomes including one-month mortality, which emphasizes the concept's potential robustness in predicting adverse events. Other studies (e.g. reference 26) have pointed in the same direction.

We suggest that frailty should be considered in clinical decision-making as well as in registers, clinical studies and policy documents. Furthermore, there is a need for prospective studies with few exclusion criteria (randomised, controlled trials, if possible from an ethical viewpoint) to study the benefit of interventions for frail cardiovascular patients. We believe that registries should be adapted for elderly NSTEMI patients including relevant measures (i.e. frailty) and relevant comorbidities (i.e. dementia). In clinical practice, individual clinical judgment and careful evaluation of initiated interventions are of great importance for these patients.

\section{Sources of Funding}

This study was supported by a Medical Research Council of Southeast Sweden grant.

\section{Disclosures}

None.

\section{Acknowledgements}

We wish to thank Elisabeth Logander, Annica Koch, Niclas Jovander and Maria Skön for their valuable contributions. We would like to acknowledge Lena Hector for her administrative assistance and LARC for monitoring the data. 


\section{References}

1. Ekerstad N, Edberg A, Carlsson P. Characteristics of multiple-diseased elderly in Swedish hospital care and clinical guidelines: do they make evidence-based priority setting a "mission impossible"? Int J Ageing

Later Life. 2009;3:71-95.

2. The Swedish National Board of Health and Welfare. Patienter i specialiserad vård 2007: Report 2009 [in Swedish]. Stockholm, Sweden: National Board of Health and Welfare; 2009.

3. American Heart Association Council. Acute coronary care in the elderly, part 1. Circulation. 2007;115:2549 2569.

4. Metha SR, Cannon CP, Fox KA, Wallentin L, Braunwald E, Yusuf S. Routine vs selective invasive strategies in patients with acute coronary. JAMA. 2005;293:2908 -2917.

5. Hoenig MR, Doust JA, Aroney CN, Scott IA. Early invasive versus conservative strategies for unstable angina and non-ST-elevation myocardial infarction in the stent era. Cochrane Database Syst Rev. 2006;(3): CD004815. doi:10.1002/14651858.CD004815.pub2.

6. Tinetti ME, Bogardus ST Jr, Agostini JV. Potential pitfalls of diseasespecific guidelines for patients with multiple conditions. N Engl J Med. 2004;351:2870 -2874.

7. Rothwell PM. External validity of randomised controlled trials: "to whom do the results of this trial apply?" Lancet. 2005;365:82-93.

8. Fortin M, Dionne J, Pinho G, Gignac J, Almirall J, Lapointe L. Randomized clinical trials: do they have external validity for patients with multiple comorbidities? Ann Fam Med. 2006;4:104 -108.

9. Lichtman JH, Spertus JA, Reid KJ, Radford MJ, Rumsfeld JS, Allen NB, Masoudi FA, Weintraub WS, Krumholz H. Acute noncardiac conditions and in-hospital mortality in patients with acute myocardial infarction. Circulation. 2007;116:1925-1930.

10. Singh M, Rihal CS, Roger VL, Lennon RJ, Spertus J, Jahangir A, Holmes DR. Comorbid conditions and outcomes after percutaneous coronary intervention. Heart. 2008;94:1424-1428

11. Fitchett D, Rockwood K. Introduction: heart disease in an elderly population. Paper presented at: Canadian Cardiovascular Society Consensus Conference; October 26-30, 2002; Toronto, Ontario, Canada.

12. National Institute for Health and Clinical Excellence. 2010. Unstable angina and NSTEMI: the early management of unstable angina and non-ST-segment-elevation myocardial infarction [CG94]. London.

13. Boyd CM, Darer J, Boult C, Fried LP, Boult L, Wu AW. Clinical practice guidelines and quality of care for older patients with multiple comorbid diseases: implications for pay for performance. JAMA. 2005;294: 716-724.

14. Smith AK, Williams BA, Lo B. Discussing overall prognosis with the very elderly. NEJM. 2011;365:21492151.

15. National Board of Health and Welfare. The Swedish National Board of Health and Welfare's guidelines for the care of heart disease 2008 [in Swedish]. http://www.socialstyrelsen.se. Accessed October 10, 2010.

16. Mitnitski A, Graham JJ, Mogilner AE, Rockwood K. Frailty, fitness and late-life mortality in relation to chronological and biological age. BMC Geriatr. 2002;2:1.

17. Rockwood K, Song X, MacKnight C, Bergman H, Hogan DB, McDowell I, Mitnitski A. A global clinical measure of fitness and frailty in elderly people. CMAJ. 2005;173:489-495. 
18. Rockwood K, Mitnitski A, Song X, Steen B, Skoog I. Long-term risks of death and institutionalization of elderly people in relation to deficit accumulation at age 70. J Am Geriatr Soc. 2006;54:975-979.

19. Fried LP, Tangen CM, Walston J, Newman AB, Hirsch C, Gottdiener J, Seeman T, Tracy R, Kop WJ, Burke G, McBurnie MA; Cardiovascular Health Study Collaborative Research Group. Frailty in older adults: evidence for a phenotype. J Gerontol A Biol Sci Med Sci. 2001;56: 146-156.

20. Hubbard RE, O’Mahony MS, Woodhouse KW. Characterising frailty in the clinical setting: a comparison of different approaches. Age Ageing. 2009;38:115-119.

21. Abellan van Kan G, Rolland Y, Houles M. The assessment of frailty in older adults. Clin Geriatr Med. 2010 May;26(2):275-86.

22. Sternberg SA, Wershof Schwartz A, Karunananthan S, Bergman H, Mark Clarfield A. The identification of frailty: a systematic literature review. J Am Geriatr Soc. 2011 Nov;59(11):2129-38.

23. Singh M, Alexander K, Roger LV, Rihal CS, Whitson HE, Lerman A, Jahangir A, Nair KS. Frailty and its potential relevance to cardiovascular care. Mayo Clin Proc. 2008;83:1146 -1153. doi:10.4065/83.10.1146.

24. Lee DH, Buth KJ, Martin B-J, Yip AM, Hirsch GM. Frail patients are at increased risk for mortality and prolonged institutional care after cardiac care. Circulation. 2010;121:973-978.

25. Murad K, Kitzman DW. Frailty and multiple comorbidities in the elderly patient with heart failure: implications for management. Heart Fail Rev 2011; Published online 31 May DOI 10.1007/s10741-011-9258-y

26. Singh M, Rihal CS, Lennon RJ. Influence of frailty and health status on outcomes in patients with coronary disease undergoing percutaneous revascularization. Circ Cardiovasc Qual Outcomes. 2011 Sep;4(5):496-502.

27. Afilalo J. Frailty in patients with cardiovascular disease: Why, when, and how to measure. Curr Cardiovasc Risk Rep. 2011 Oct;5(5):467-72.

28. Myers V, Drory Y, Gerber Y. Clinical relevance of frailty trajectory post myocardial infarction. European Journal of Preventive Cardiology published online 1 October 2012 DOI:10.1177/2047487312462828.

29. Sachdev M, Sun JL, Tsiatis AA, Nelson CL, Mark DB, Jollis JG. The prognostic importance of comorbidity for mortality in patients with stable coronary artery disease. J Am Coll Cardiol. 2004;43:576 -582.

30. Ekerstad N, Swahn E, Janzon M, Alfredsson J, Löfmark R, Lindenberger M, Carlsson P. 2011. Frailty Is Independently Associated With Short-Term Outcomes for Elderly patients With Non-ST-Segment Elevation Myocardial Infarction. Circulation 2011;124:2397-2404.

31. Shrout PE, Fleiss JL. Intraclass correlations: uses in assessing rater reliability. Psychol Bull. 1979;86:420428.

32. Burdock EI, Fleiss JL, Hardesty AS. A new view of interobserver agreement. Personnel Psychol. $1963 ; 16: 373-384$.

33. Lagerqvist B, Husted S. Kontny F, Ståhle E, Swahn E, Wallentin L. 5-year outcomes in the FRISC-II randomised trial of an invasive versus a non-invasive strategy in non-ST-elevation acute coronary syndrome: a follow-up study. Lancet. 2006;368:998-1004.

34. Kastrati A, Neumann FJ, Mehilli J, Byrne RA, Iijima R, Bu“ttner HJ, Khattab AA, Schulz S, Blankenship JC, Pache J, Minners J, Seyfarth M, Graf I, Skelding KA, Dirschinger J, Richardt G, Berger PB, Scho"mig A; ISAR-REACT 3 Trial Investigators. Bivalirudin versus unfractionated heparin during percutaneous coronary intervention. N Engl J Med. 2008; 359:688-696.

35. The Swedish National Board of Health and Welfare. Swedish national guidelines for stroke care 2005. http://www.socialstyrelsen.se/guidelines/ national. Accessed February 3, 2009. 
36. Thygesen K, Alpert JS, White HD. Joint ESC/ACCF/AHA/WHF Task Force for the Redefinition of Myocardial Infarction. Universal definition of myocardial infarction. Circulation. 2007;116:2634 -2653.

37. Cox DR. Regression models and life-tables. Journal of the Royal Statistical Society Series B (Methodological). 1972;34:187-220.

38. Allison PD. Logistic Regression Using the SAS System: Theory and Application. Cary, NC: Wiley-SAS; 2001.

39. Purser JL, Kuchibhatla MN, Fillenbaum GG, Harding TH, Peterson ED, Alexander KP. Identifying frailty in hospitalized older adults with significant coronary artery disease. J Am Geriatr Soc. 2006;54: 1674 -1681.

40. Fried LP, Ferrucci L, Darer L, Williamson JD, Anderson G. Untangling the concepts of disability, frailty, and comorbidity: implications for improved targeting and care. J Gerontol A Biol Sci Med Sci. 2004;59:255-263.

41. Levey AS, Eckardt KU, Tsukamoto Y, Levin A, Coresh J, Rossert J, De Zeeuw D, Hostetter TH, Lameire N, Eknoyan G. Definition and classification of chronic kidney disease: a position statement from Kidney

Disease: Improving Global Outcomes (KDIGO). Kidney Int. 2005;67: 2089-2100. 
Figure 1. The Canadian Study of Health and Aging (CSHA) Clinical Frailty Scale (CFS)

1 Very fit - robust, active, energetic, well motivated and fit; these people commonly exercise regularly and are in the most fit group for their age

2 Well - without active disease, but less fit than people in category 1.

3 Well, with treated comorbid disease - disease symptoms are well controlled compared with those in category 4

4 Apparently vulnerable - although not frankly dependent, these people commonly complain of being "slowed up" or have disease symptoms.

5 Mildly frail - with limited dependence on others for instrumental activities of daily living

6 Moderately frail - help is needed with both instrumental and noninstrumental activities of daily living

7 Severely frail - completely dependent on others for the activities of daily living, or terminally ill.

(Rockwood, K. et al. (2005). A global clinical measure of fitness and frailty in elderly people. CMAJ 173(5): 489-95.) 
Figure 2. The CAD-specific index (Sachdev 2004). The integer coefficients are summed up and the resulting score denotes risk group: 0-1 low risk; 2-3 moderate risk; and =>4 high risk.

\begin{tabular}{ll}
\hline Risk factor & Integer coefficient \\
\hline Current smoker & 1 \\
Hypertension & 1 \\
History of CVA/TIA & 1 \\
DM & 2 \\
DM with sequelae & 3 \\
COPD & 2 \\
PVD & 2 \\
Tumour/lymphoma/leukemia & 2 \\
Moderate to severe renal disease* & 7 \\
Metastatic cancer & 5 \\
\hline
\end{tabular}

* Defined as creatinine $>3 \mathrm{mg} / \mathrm{dl}(29)$ 
Figure 3. The proportion of patients in each frailty stage not undergoing coronary angiography during the index episode of care. The figures denote numbers of patients.

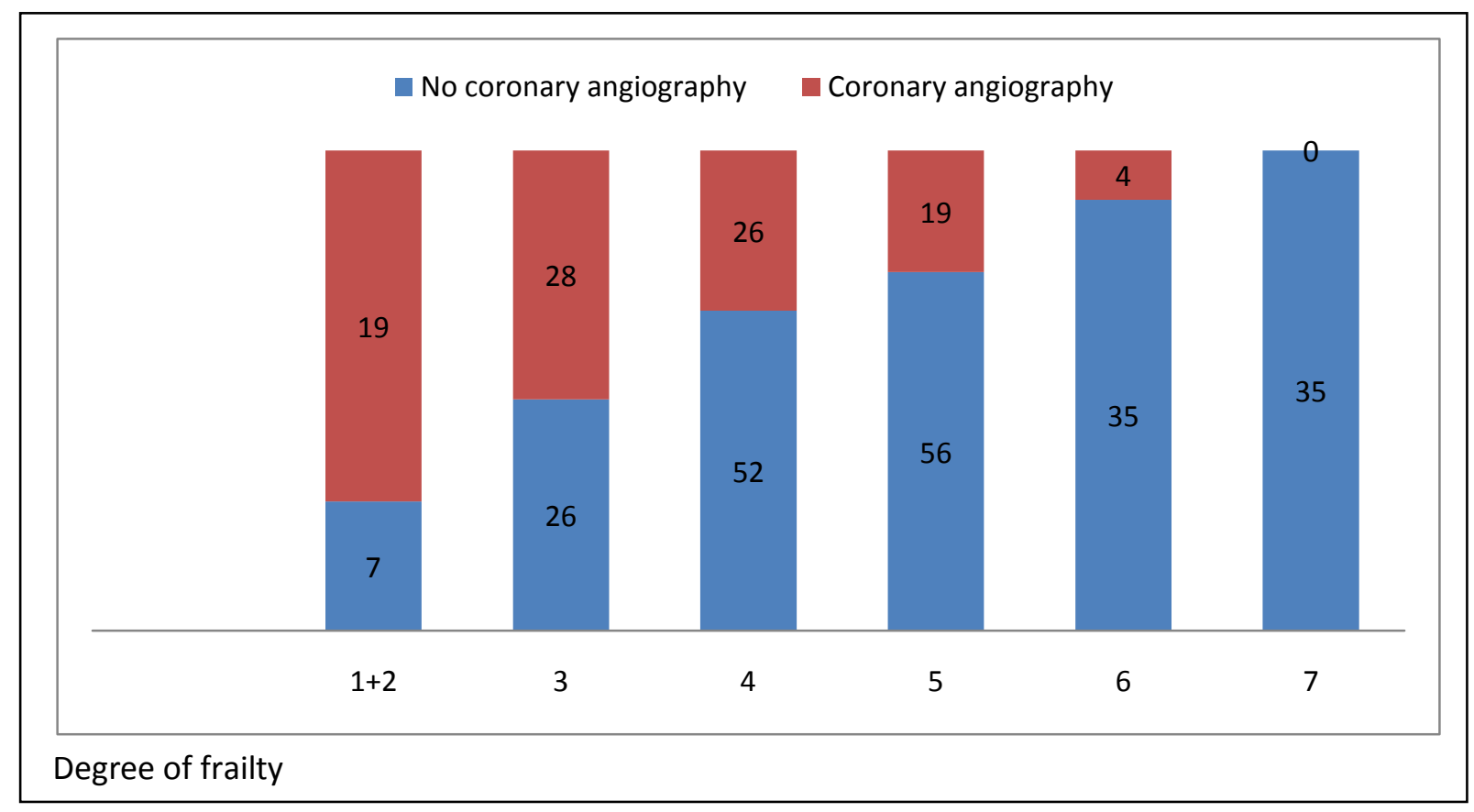


Table 1 Baseline patient characteristics.

\begin{tabular}{|c|c|c|c|}
\hline Variable, n (\%) & $\begin{array}{l}\text { Non-frail } \\
(n=158)\end{array}$ & $\begin{array}{l}\text { Frail } \\
(n=149)\end{array}$ & $\mathrm{P}$ \\
\hline Mean age, years\# & 83 & 85 & 0.0003 \\
\hline Female sex & $69(43.7)$ & $81(54.4)$ & 0.068 \\
\hline Diabetes & $29(18.4)$ & $41(27.5)$ & 0.058 \\
\hline COPD & $8(5.1)$ & $19(12.8)$ & 0.025 \\
\hline $\mathrm{CHF}$ & $25(15.8)$ & $56(37.6)$ & $<0.0001$ \\
\hline Severe renal impairment* & $26(16.5)$ & $56(37.6)$ & $<0.0001$ \\
\hline PVD & $9(5.7)$ & $18(12.1)$ & 0.068 \\
\hline CVD & $26(16.5)$ & $50(33.6)$ & 0.0006 \\
\hline Dementia & $9(5.7)$ & $41(27.5)$ & $<0.0001$ \\
\hline Anemia & $51(32.3)$ & $84(56.4)$ & $<0.0001$ \\
\hline Malignant disease & $20(12.7)$ & $25(16.8)$ & 0.336 \\
\hline Previous myocardial infarction & $59(37.3)$ & $70(47.0)$ & 0.106 \\
\hline Type 2 myocardial infarction $\S$ & $50(31.7)$ & $56(37.6)$ & 0.283 \\
\hline Medium or high cardio- & $136(86.1)$ & $140(94.0)$ & 0.024 \\
\hline \multicolumn{4}{|l|}{ vascular risk } \\
\hline High CAD-index score & $36(22.8)$ & $62(41.6)$ & 0.0006 \\
\hline Highest troponin & 1.1 & 1.4 & 0.43 \\
\hline Lowest $\mathrm{Hb}$ & 133 & 116 & 0.028 \\
\hline Highest creatinine & 117 & 148 & 0.002 \\
\hline Highest glucose & 8.4 & 11.3 & 0.055 \\
\hline Highest CRP & 26 & 71 & $<.0001$ \\
\hline
\end{tabular}

COPD indicates chronic obstructive pulmonary disease; CAD, coronary artery disease.

*Defined as glomerular filtration rate <30 (41)

$\S$ Myocardial infarction secondary to ischemia due to either increased $\mathrm{O}_{2}$

demand or decreased supply (eg, coronary artery spasm, coronary embolism,

anemia, arrhythmias, hypertension, or hypotension). (36)

\# Age range non-frail: 75-102 years; frail 75-101 years

Laboratory data are reported as mean values. 
Table 2. Unadjusted outcomes, one year

\begin{tabular}{|c|c|c|c|}
\hline & onfrail $(n=158)$ & Frail $(n=149)$ & $\mathrm{P}$ \\
\hline Mortality & $20(12.7)$ & $73(49.0)$ & $<.0001$ \\
\hline \multicolumn{4}{|l|}{ One-year } \\
\hline \multicolumn{4}{|l|}{ composite } \\
\hline outcome* & 109 (69.0) & $122(81.9)$ & 0.011 \\
\hline Reinfarction & $29(18.4)$ & $33(22.1)$ & 0.48 \\
\hline \multicolumn{4}{|l|}{ Major bleeding, } \\
\hline \multicolumn{4}{|l|}{ Stroke/TIA or } \\
\hline need for dialysis & $23(14.6)$ & $19(12.8)$ & 0.74 \\
\hline Rehospitalization & 107 (67.7) & $92(61.7)$ & 0.28 \\
\hline
\end{tabular}

*One-year composite outcome denotes the composite of death from any cause, myocardial reinfarction, revascularization due to ischemia, hospitalization for any cause, major bleeding, stroke/transient ischemic attack (TIA), and need for dialysis up to one year after inclusion. 
Table 3 Adjusted mortality.

\begin{tabular}{|c|c|c|c|c|}
\hline & \multicolumn{2}{|l|}{ 0-30 days } & \multicolumn{2}{|l|}{ 31-365 days } \\
\hline & HR (CI) & $\mathbf{P}$ & HR (CI) & $\mathbf{P}$ \\
\hline Age & & 0,1288 & & 0,0354 \\
\hline $75-79$ & REF & & REF & \\
\hline $80-84$ & $0,88(0,25-3,17)$ & 0,8483 & $1,79(0,82-3,90)$ & 0,1430 \\
\hline$\geq 85$ & $2,20(0,75-6,45)$ & 0,1531 & $2,66(1,25-5,69)$ & 0,0114 \\
\hline Gender & & 0,4605 & & 0,4688 \\
\hline Male & $\mathrm{REF}$ & & REF & \\
\hline Female & $0,73(0,32-1,67)$ & 0,4605 & $1,23(0,70-2,14)$ & 0,4688 \\
\hline Ejection faction & & 0,1558 & & 0,0195 \\
\hline$>40 \%$ & $\mathrm{REF}$ & & $\mathrm{REF}$ & \\
\hline$\leq 40 \%$ & $2,44(0,82-7,22)$ & 0,1083 & $2,47(1,27-4,80)$ & 0,0076 \\
\hline Not recorded & $2,53(0,93-6,89)$ & 0,0688 & $1,94(1,05-3,57)$ & 0,0340 \\
\hline Cardiovascular risk & & 0,6367 & & 0,0155 \\
\hline Low & $\mathrm{REF}$ & & $\mathrm{REF}$ & \\
\hline Moderate & $2,71(0,35-21,33)$ & 0,3429 & $2,52(0,76-8,36)$ & 0,1305 \\
\hline Severe & $2,57(0,29-22,80)$ & 0,3969 & $5,38(1,45-19,96)$ & 0,0120 \\
\hline Frailty & & 0,0164 & & $<, 0001$ \\
\hline Nonfrail & REF & & REF & \\
\hline Frail & $3,43(1,25-9,36)$ & 0,0164 & $4,33(2,41-7,78)$ & $<, 0001$ \\
\hline CAD index & & 0,0352 & & 0,2141 \\
\hline Low & $\mathrm{REF}$ & & REF & \\
\hline Moderate & $0,64(0,17-2,43)$ & 0,5125 & $0,76(0,40-1,43)$ & 0,3927 \\
\hline Severe & $2,44(1,01-5,89)$ & 0,0467 & $0,57(0,31-1,07)$ & 0,0813 \\
\hline
\end{tabular}

CI indicates confidence interval; CAD, coronary artery disease. The independent variables were tested for collinearity with the use of the variance inflation factor. All variables had a variance inflation factor value _2.5, which does not indicate collinearity. 
Figure 4. Survival analysis, unadjusted.

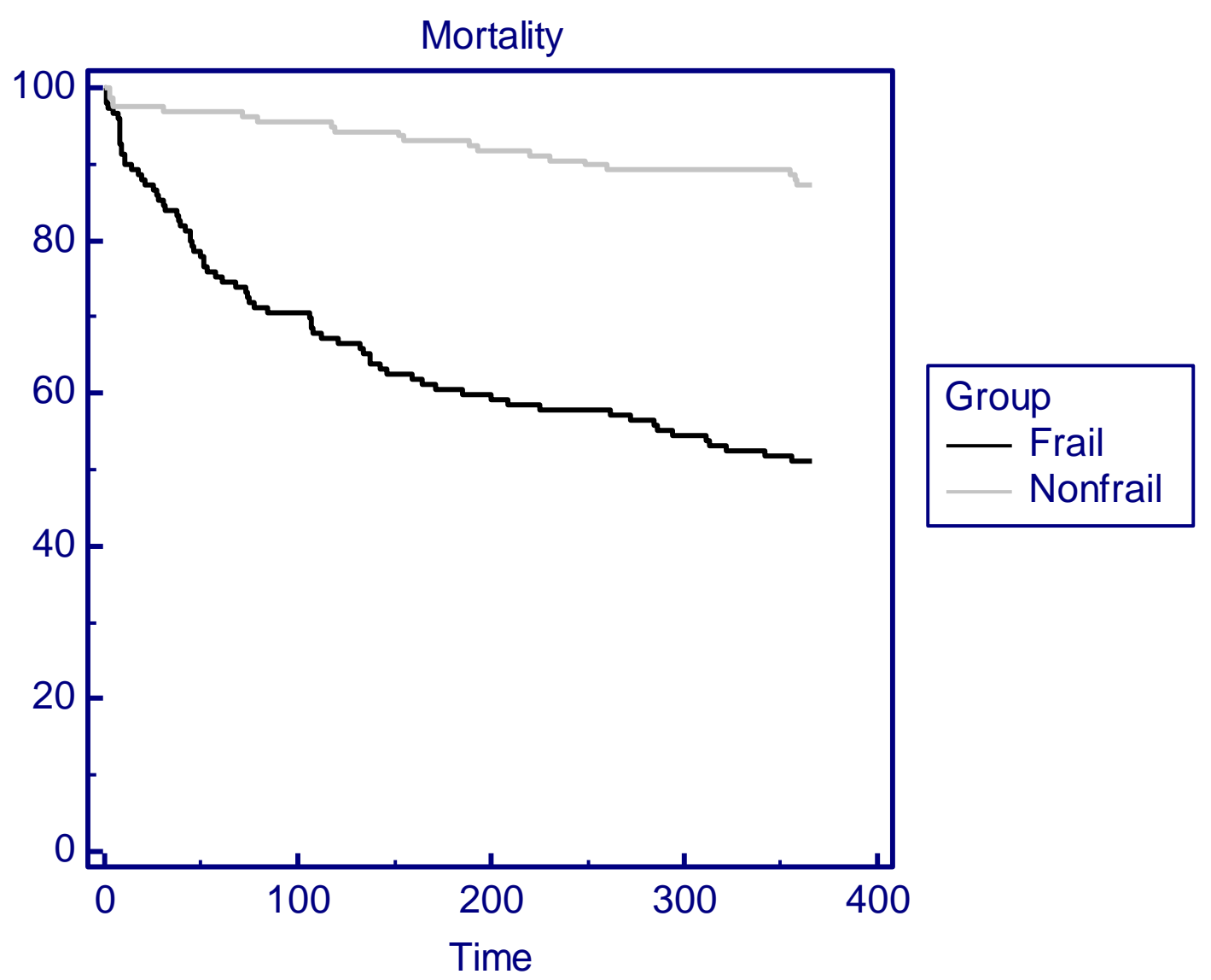


